\title{
Safety and applicability of a pre-stage public access ventilator for trained laypersons: a proof of principle study
}

Patricia Fuchs ${ }^{1 *}$ (D), Juliane Obermeier ${ }^{1}$, Svend Kamysek ${ }^{1}$, Martin Degner ${ }^{2}$, Hannes Nierath ${ }^{2}$, Henning Jürß ${ }^{2}$, Hartmut Ewald ${ }^{2}$, Jens Schwarz ${ }^{3}$, Martin Becker ${ }^{3}$ and Jochen K. Schubert ${ }^{1}$

\begin{abstract}
Background: Contemporary resuscitation guidelines for basic life support recommend an immediate onset of cardiac compressions in case of cardiac arrest followed by rescue breaths. Effective ventilation is often omitted due to fear of doing harm and fear of infectious diseases. In order to improve ventilation a pre-stage of an automatic respirator was developed for use by laypersons.

Methods: Fifty-two healthy volunteers were ventilated by means of a prototype respirator via a full-face mask in a pilot study. The pre-stage public access ventilator (PAV) consisted of a low-cost self-designed turbine, with sensors for differential pressure, flow, $\mathrm{FO}_{2}, \mathrm{FCO}_{2}$ and 3-axis acceleration measurement. Sensor outputs were used to control the respirator and to recognize conditions relevant for efficiency of ventilation and patients' safety. Different respiratory manoeuvres were applied: a) pressure controlled ventilation (PCV), b) PCV with controlled leakage and c) PCV with simulated airway occlusion. Sensor signals were analysed to detect leakage and airway occlusion. Detection based upon sensor signals was compared with evaluation based on clinical observation and additional parameters such as exhaled $\mathrm{CO}_{2}$.

Results: Pressure controlled ventilation could be realized in all volunteers. Leakage was recognized with 93.5\% sensitivity and $93.5 \%$ specificity. Simulated airway occlusion was detected with $91.8 \%$ sensitivity and $91.7 \%$ specificity.

Conclusion: The pre-stage PAV was able to detect potential complications relevant for patients' safety such as leakage and airway occlusion in a proof of principle study. Prospectively, this device provides a respectable basis for the development of an automatic emergency respirator and may help to improve bystander resuscitation.
\end{abstract}

Keywords: Prototype respirator, Pressure controlled ventilation, Airway occlusion, Lay resuscitation

\section{Background}

Sudden cardiac arrest is one of the major causes of death in Europe [1, 2]. Survival and neurological favourable outcome after cardiac arrest depend on several factors like early recognition, immediate bystander cardio-pulmonary resuscitation (CPR) and high quality CPR including effective chest compressions. Successful resuscitation in case of ventricular fibrillation (VF) cardiac arrest is facilitated by early electrical defibrillation $[3,4]$.

\footnotetext{
*Correspondence: patricia.fuchs@uni-rostock.de

${ }^{1}$ Department of Anaesthesiology and Intensive Care Medicine, Rostock University Medical Centre, Schillingallee 35, 18057 Rostock, Germany Full list of author information is available at the end of the article
}

The importance of oxygenation and ventilation during lay CPR has been discussed controversially over years [5-7]. During respiratory and cardiac arrest ventilation and circulation and thereby oxygen delivery is interrupted. Continued cell metabolism and oxygen consumption leads to decreased oxygen content and critical oxygen supply for vital organs. Oxygen supply for brain and heart is an important variable that determines success probability of cardio-pulmonary resuscitation in cardiac arrest especially of hypoxic origin and during prolonged CPR. Additionally, ventilation is important for carbon dioxide elimination and cardio-pulmonary arrest thus results in respiratory acidosis. Focus of the new 
ERC guidelines (European Resuscitation Council) for basic life support (BLS) published in 2015 again is the recommendation of an immediate initiation of cardiac compressions by laypersons in case of out of hospital cardiac arrest [4]. Trained CPR providers should perform chest compressions combined with additional rescue breaths. From a pathophysiological point of view, oxygenation and ventilation remain essential to improve outcomes particularly in cardiac arrest of non-cardiac respectively respiratory origin or prolonged resuscitation efforts. Rescue breaths as well as chest compressions are critical for successful resuscitation in these cases [4].

However, $53 \%$ of cardiac arrests were in fact witnessed, but only $32 \%$ of witnesses performed conventional CPR as reported by Sasson [8]. Possible reasons to withhold resuscitation efforts could be constraints of laypersons to do harm or fear of infectious diseases by performing mouth-to-mouth ventilation [9]. From different studies, it is also known that the effectiveness of ventilation by laypersons is significantly reduced if the training dates back long time ago. Therefore, if willingness to ventilation by laypersons does not exist chest compressions only are recommended $[10,11]$.

Unlike public access automated external defibrillators (AEDs) or treatment of VF, respirators for effective and safe ventilation are not yet available for use by laypersons during CPR. The concept of public access defibrillators might serve as an exemplary, successful model for life-saving technology applied by laypersons. Early defibrillation can be achieved through CPR providers using AEDs which have already been installed in many public institutions or places. These AEDs can be used safely both by trained laypersons and professional first aid responders [3, 12].

The intention of this project was to develop an automated ventilator in order to support laypersons, who are willing to perform rescue ventilation in patients with sudden cardiac arrest. The functional prototype of the automated public access ventilator (PAV) was conceived as a turbine combined with smart sensor technology. For patients' safety, reliable detection of malfunction, leakage and airway occlusion is mandatory. Therefore, safety and applicability of the newly developed pre-stage ventilator were evaluated as a first step in healthy volunteers in this proof of principle study.

\section{Methods}

\section{Study design and participants}

This study was designed as a single-centre observational study and carried out in accordance with the 'Declaration of Helsinki'. After approval of the ethics committee from Rostock University Medical Centre (approval $\mathrm{N}^{\circ}$ : A 2014-0015) and after having obtained written informed consent, 52 healthy volunteers were enrolled into the study. Demographic data from 52 healthy volunteers (28 males, 24 females; aged between 20 and 58 years) are shown in Table 1.

\section{Prototype setting}

The pre-stage PAV consisted of a low-cost self-designed turbine and sensors (mainly developed by Sensatronic $\mathrm{GmbH}$ ) for measuring differential pressure, flow, 3-axis acceleration together with mainstream $\mathrm{FO}_{2}$ (fraction of oxygen) and $\mathrm{FCO}_{2}$ (fraction of carbon dioxide) with high time resolution (Fig. 1). The turbine was equipped with a brushless motor and works with a fast pressure directed closed loop control to realize respiration profiles without mechanical valves. The maximal turbine flow was $130 \mathrm{l} / \mathrm{min}$ at a power consumption of $38 \mathrm{~W}$. The sensors used were based on following measuring principles:

- Flow: anemometric

- Pressure: piezoresistive

- $\mathrm{FO}_{2}$ : electrochemical

- $\mathrm{FCO}_{2}$ : non-dispersive infrared absorption (NDIR)

- Position: 3-acis accelerometer (micro electro mechanical system, MEMS).

The combination of different sensors in the pre-stage PAV prototype induced some additional dead space. As $\mathrm{PCO}_{2}$ remained constant, this additional dead space was not relevant for ventilation.

A well-directed control of the pre-stage PAV is based on the combination of multiple sensor data and realized by a stand-alone sbRIO-9632 controller board (National Instruments Inc., Munich, Germany). The hardware adaption, signal acquisition and data analysis as well as the complex control of the respirator were carried out by the Institute for General Electrical Engineering of the University of Rostock.

\section{Experimental protocol}

The measurements were performed after the volunteers rested for $15 \mathrm{~min}$, while they received instructions by the tutor. All volunteers were placed in a supine position on a blanket and were ventilated by means of the prototype respirator via a full-face mask with fixation (Ultra Mirage, ResMed GmbH \& Co. KG, Germany) connected

Table 1 Demographic characteristics of healthy volunteers

\begin{tabular}{llll}
\hline & Total & Men & Women \\
\hline Number $(n, \%)$ & 52 & $28(54)$ & $24(46)$ \\
Age [years] (mean \pm SD) & $31.9 \pm 9.5$ & $32.6 \pm 10.0$ & $31.0 \pm 8.7$ \\
Height [cm] (mean \pm SD) & $176.1 \pm 9.6$ & $181.6 \pm 8.0$ & $169.6 \pm 7.0$ \\
Weight [kg] (mean \pm SD) & $72.4 \pm 14.3$ & $80.0 \pm 13.3$ & $63.6 \pm 9.5$ \\
BMI (mean \pm SD) & $23.2 \pm 3.1$ & $24.2 \pm 3.3$ & $22.1 \pm 2.4$ \\
\hline
\end{tabular}

$S D$ standard deviation, $B M I$ body mass index 


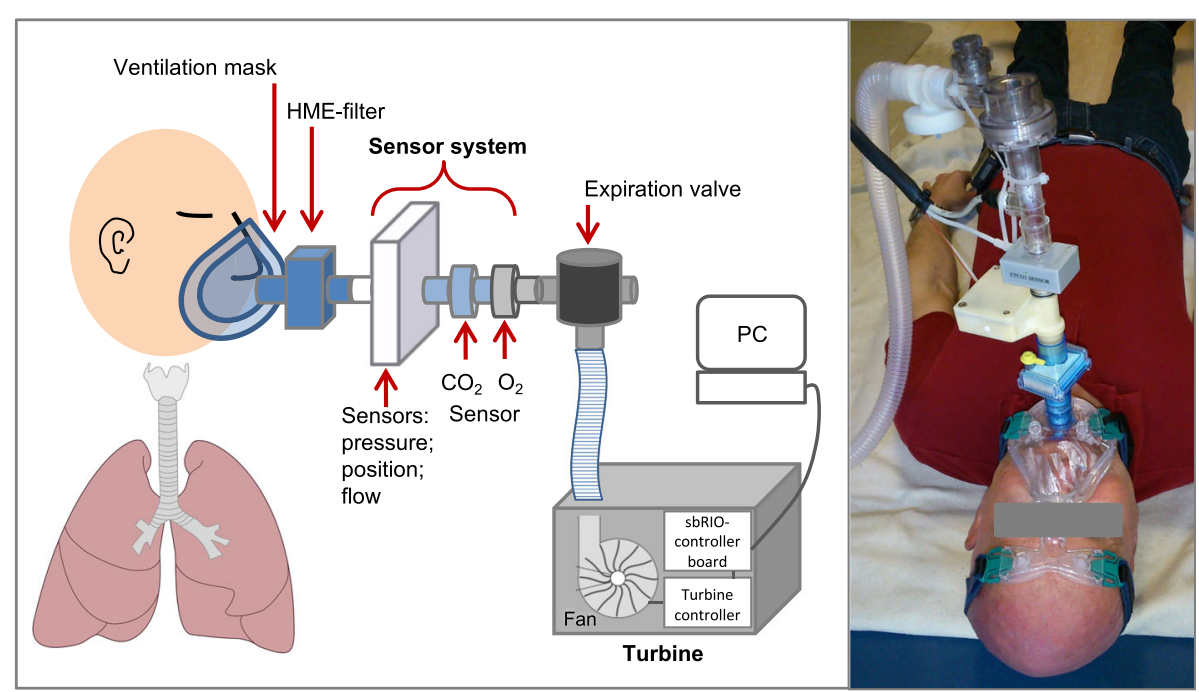

Fig. 1 Schematic diagram of sensor prototype and photograph of experimental setup

to the sensor system. Medium and large size masks were used for women and men according to the optimal facial fit. To ensure volunteers' safety the experimental setting was observed by a physician. Pressure controlled ventilation was performed with inspiratory pressure ( $\mathrm{p}_{\text {insp }}$ ) $1.5 \mathrm{kPa}$, positive end expiratory pressure (PEEP) $0 \mathrm{kPa}$, respiratory rate (RR) 10 breaths $/ \mathrm{min}, \mathrm{I}: \mathrm{E}=1: 2$, and inspiratory oxygen fraction $\left(\mathrm{F}_{\mathrm{i}} \mathrm{O}_{2}\right) 0.21$.

In order to evaluate the safety and applicability of the pre-stage PAV, three different respiratory manoeuvres were applied for the duration of $1 \mathrm{~min}$, respectively:

- Pressure controlled ventilation (PCV)

- PCV with controlled leakage

- PCV with simulated airway occlusion

During a second study phase these three respiratory manoeuvres were performed under reduced lung compliance induced by wrist weights $(14 \mathrm{~kg})$ placed on the chest.

Pressure controlled ventilation was performed after instructing the participants to stop breathing spontaneously and to tolerate ventilation.

For performance of a reproducible leakage manoeuvre, two mask ports $\left(7 \mathrm{~mm}^{2}\right.$ each) were opened after $2-3$ ventilations, remained open during the following 5 ventilations and were closed again subsequently. The airway occlusion manoeuvre was performed after a resting phase of $5 \mathrm{~min}$. After 2-3 ventilations the participants were asked to close their upper-airway actively for 3 ventilations and to open the airway again afterwards.

\section{Data acquisition and statistical analysis}

During ventilation manoeuvres all raw data as well as the analysed results were logged continuously by the
sbRIO-9632 based prototype device. Data analysis and visualization were realized by means of a MatLab-based algorithm established by the Institute for General Electrical Engineering.

Features based on time-resolved integrations of flow and pressure values were used to detect leakage and airway occlusion, respectively. These complications were detected for each breath by the sensor based algorithms of the PAV prototype. Comparisons were done with the evaluation based on clinical observation considering multi-parameter assessment such as adequate generation of flow and pressure as well as exhaled $\mathrm{O}_{2}$ and $\mathrm{CO}_{2}$ signals.

The criteria for detection of leakage and airway occlusion are given in Table 2.

Statistical analysis was performed with SIGMAPLOT 10 (Systat Software GmbH, Erkrath, Germany). Receiver operating characteristics (ROC) were generated to

Table 2 Criteria for detection of different manoeuvres

\begin{tabular}{|c|c|c|}
\hline & $\begin{array}{l}\text { Mathematical } \\
\text { algorithm }\end{array}$ & Clinical observation \\
\hline \multirow[t]{4}{*}{ Leakage } & \multirow{4}{*}{$\begin{array}{l}\text { Integration (time) } \\
\text { of expiratory and } \\
\text { inspiratory flow } \\
\text { for volume } \\
\text { estimation } \\
\left(V_{\exp } \text { and } V_{\text {insp }}\right)\end{array}$} & Curve characteristics: \\
\hline & & $V_{\text {insp }}>V_{\text {exp }}$ \\
\hline & & $p_{\text {eff }} \neq p_{\text {insp }}$ \\
\hline & & $\mathrm{Flow}_{\text {insp }}>50 \mathrm{l} / \mathrm{min}$ \\
\hline \multirow[t]{4}{*}{ Airway occlusion } & \multirow{4}{*}{$\begin{array}{l}\text { Integration (time) } \\
\text { of expiratory and } \\
\text { inspiratory } \\
\text { pressure }\end{array}$} & Curve characteristics: \\
\hline & & $\mathrm{ETCO}_{2} \leq 2 \% ; \mathrm{FO}_{2}=$ constant \\
\hline & & $p_{\text {eff }}=p_{\text {insp }}$ \\
\hline & & Flow $\ll 10 \mathrm{l} / \mathrm{min}$ \\
\hline
\end{tabular}

$V_{\text {exp }}$ expiratory volume, $V_{\text {insp }}$ inspiratory volume, $E T C O_{2}$ end tidal fraction of carbon dioxide, $\mathrm{FO}_{2}$ oxygen fraction, $p_{\text {eff }}$ effective pressure, $p_{\text {insp }}=$ inspiratory pressure, Flow $_{\text {insp }}=$ inspiratory flow 
estimate the sensitivity and specificity of the PAV system in comparison to medical evaluations. The cut-off values were based on feature extraction from temporal integration of expiratory and inspiratory flow (for leakage) as well as from temporal integration of expiratory and inspiratory pressure (for airway occlusion).

\section{Results}

Pressure controlled ventilation was applied in all volunteers according to inspiratory pressure and respiratory rate set to $1.5 \mathrm{kPa}$ and 10 breaths $/ \mathrm{min}$, respectively. Resulting tidal volumes were in the range of $8-12 \mathrm{ml} / \mathrm{kg}$.

Figures 2, 3 and 4 show results obtained from different sensor outputs during the applied respiratory manoeuvres. The graphs illustrate flow and pressure as well as $\mathrm{FO}_{2}$ and $\mathrm{FCO}_{2}$ values over time.

Figure 2 describes pressure controlled ventilation without any intervention in one healthy volunteer characterized by typical curves for flow, pressure, $\mathrm{FO}_{2}$ and $\mathrm{FCO}_{2}$ recorded over one minute.

Figure 3 illustrates PCV with controlled leakage according to the study protocol. As a consequence during leakage expiratory flow decreased, while the $\mathrm{FO}_{2}$ and $\mathrm{FCO}_{2}$ curves did not change markedly. During the leakage manoeuvre expiratory volumes decreased compared to inspiratory volumes. Compared to ventilation without leakage the decrease of expiratory tidal volume was 25 to $35 \%$.

Figure 4 shows PCV interrupted by an airway occlusion manoeuver according to the study protocol. During upper airway occlusion, no typical in- and expiratory flow curve was generated and therefore $\mathrm{FO}_{2}$ and $\mathrm{FCO}_{2}$ remained on a constant level. The pre-set airway pressure ( $\left.\mathrm{p}_{\text {insp }}\right)$ was always reached, but never exceeded $1.5 \mathrm{kPa}$. With respect to the criteria for complete airway occlusion being defined in Table 2 (e.g. $\mathrm{ETCO}_{2} \leq 2 \%$ ), $80 \%$ of all volunteers were able to close their airways entirely.

All results from the second study phase with PCV manoeuvers under reduced lung compliance are presented in Additional file 1: Figure S1; Additional file 2: Figure S2; and Additional file 3: Figure S3.

Leakage and airway occlusion were recognized with over $92 \%$ sensitivity and specificity. Fig. 5 presents the corresponding ROC curves. Table 3 summarizes relevant values for sensitivity and specificity of the ROC curves for different manoeuvres.

\section{Discussion}

In this pilot study, we demonstrated safety and applicability of an innovative pre-stage public access ventilator. The compact PAV system consisting of a turbine, sensors (flow, pressure, $\mathrm{FO}_{2}, \mathrm{FCO}_{2}, 3$-axis accelerometer) and a control unit was able to provide pressure controlled ventilation in healthy volunteers. Detection of potential complications relevant for patient's safety such as leakage and airway occlusion was realized with sensitivity and specificity $>92 \%$.

Although only half of cardiac arrests were witnessed, layperson CPR has been shown to be an important predictor of increased survival to hospital discharge $[13,14]$. However, laypersons are often in doubt if they can perform effective ventilation. Aggravating this situation, there is a risk for gastric air insufflation resulting in a potential risk for pulmonary aspiration $[15,16]$. In the current literature, there is only a small number of publications addressing the effectivity of ventilation by laypersons
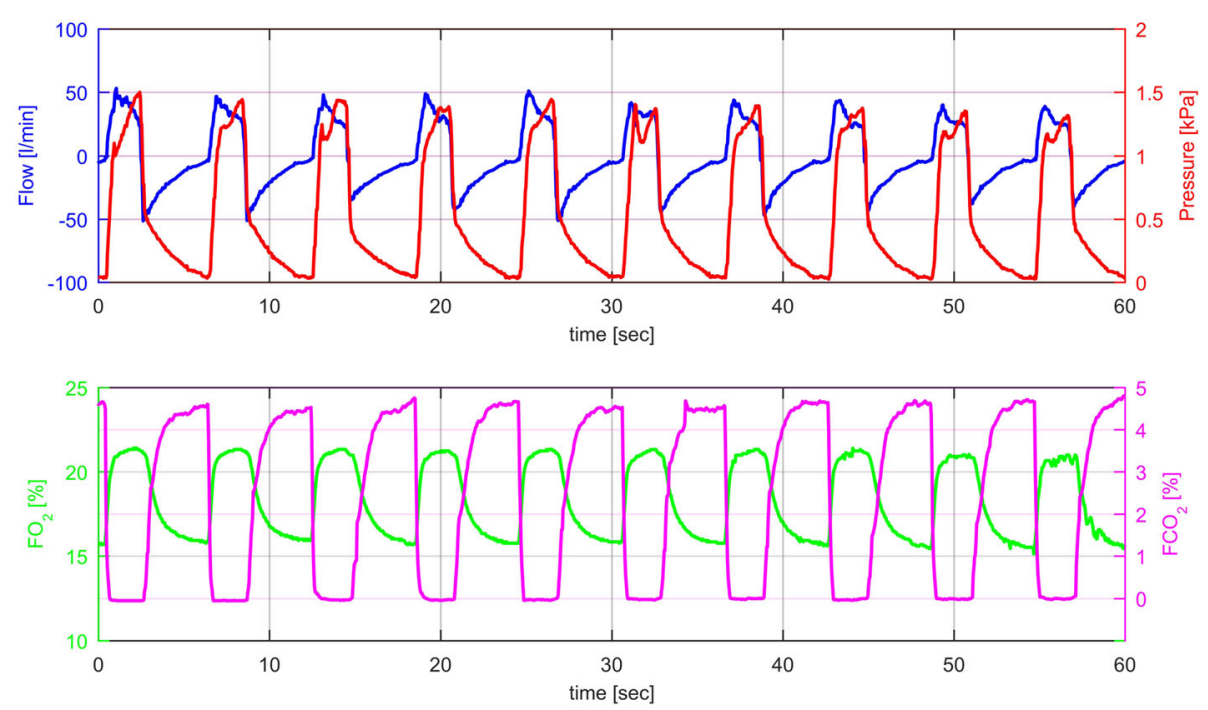

Fig. 2 Sensor outputs during PCV without any intervention in one healthy volunteer characterized by typical curves for flow, $\mathrm{Pressure}_{1} \mathrm{FO}_{2}$ and $\mathrm{FCO}_{2}$ recorded over one minute (blue line $=$ flow $[\mathrm{l} / \mathrm{min}]$; red line $=$ pressure $[\mathrm{kPa}]$; green line $=\mathrm{FO}_{2}[\%]$; magenta line $=\mathrm{FCO}_{2}[\%]$ ) 


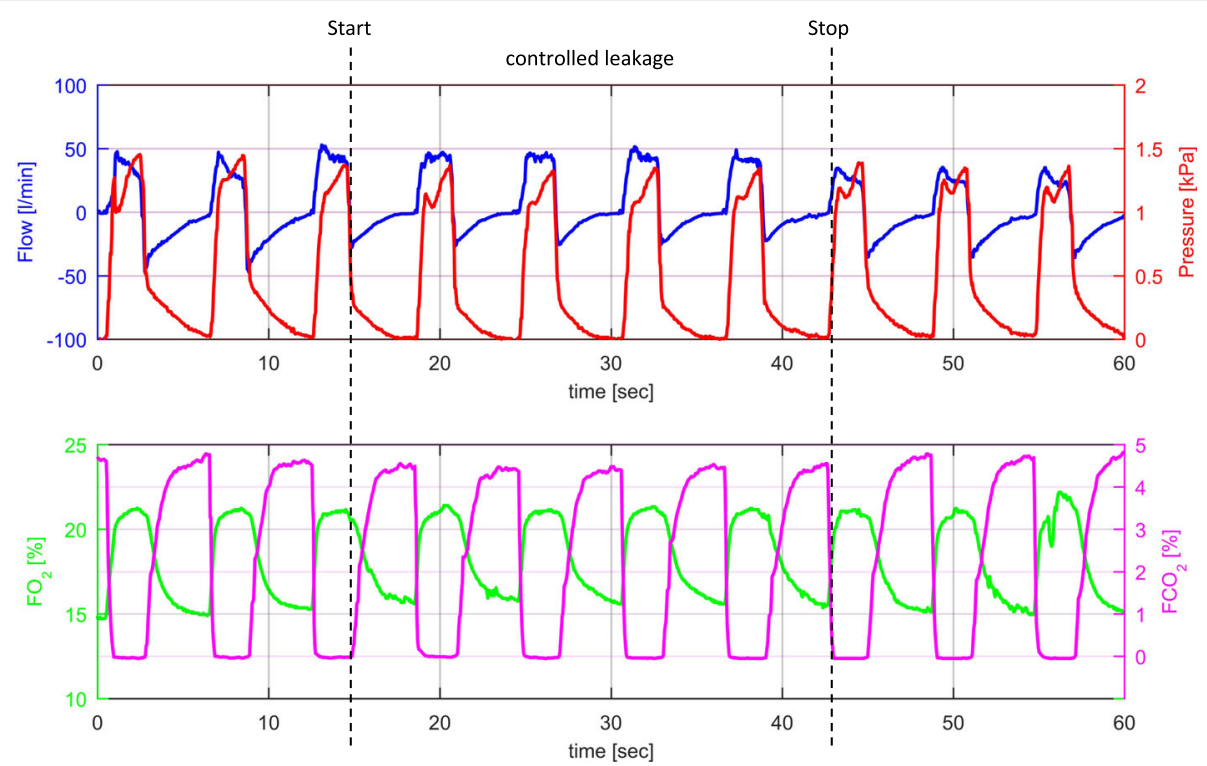

Fig. 3 Sensor outputs during PCV and controlled leakage according to the study protocol in one healthy volunteer characterized by curves for flow, pressure, $\mathrm{FO}_{2}$ and $\mathrm{FCO}_{2}$ recorded over one minute (black dashed lines = start and stop of controlled leakage; blue line = flow [l/min]; red line $=$ pressure $[\mathrm{kPa}]$; green line $=\mathrm{FO}_{2}[\%]$; magenta line $=\mathrm{FCO}_{2}[\%]$ )

[17-19]. In 2003, when the ERC guidelines recommended explicitly rescue breaths for CPR, Woollard et al. used a manikin model of cardiac arrest to compare skill performance in untrained laypersons receiving compressions-only or standard telephone CPR [17]. A number of subjects did not open the airway and more than $75 \%$ in the standard telephone group failed to deliver two effective initial rescue breaths.
Only $17 \%$ provided an adequate inflation volume for subsequent breaths. The problem of delays to first compression and poor performance of airway opening and ventilation skills was also reported by Kellermann in a simulated cardiac arrest scenario [18]. The use of a public access respirator could support trained laypersons by effective automated non-invasive ventilation during CPR.

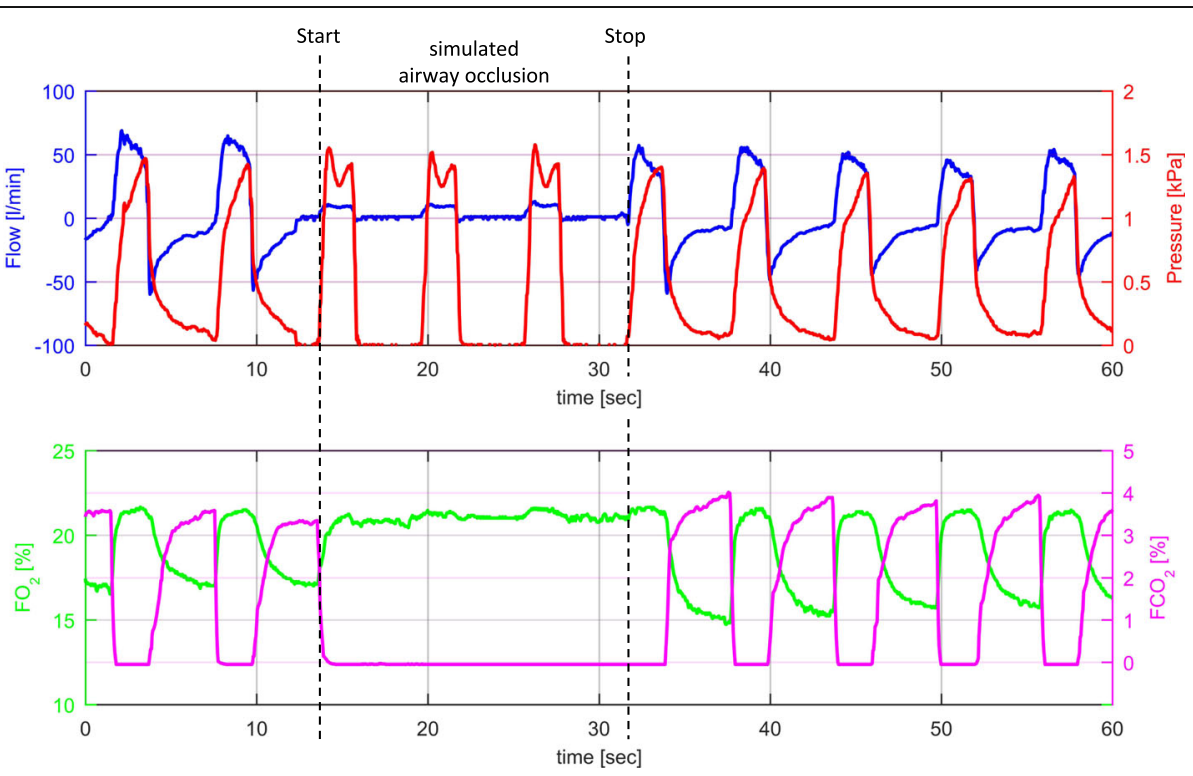

Fig. 4 Sensor outputs during PCV and simulated airway occlusion according to the study protocol in one healthy volunteer characterized by curves for flow, pressure, $\mathrm{FO}_{2}$ and $\mathrm{FCO}_{2}$ recorded over one minute (black dashed lines = start and stop of simulated airway occlusion; blue line $=$ flow $[\mathrm{l} / \mathrm{min}]$; red line $=$ pressure $[\mathrm{kPa}]$; green line $=\mathrm{FO}_{2}[\%]$; magenta line $=\mathrm{FCO}_{2}[\%]$ ) 

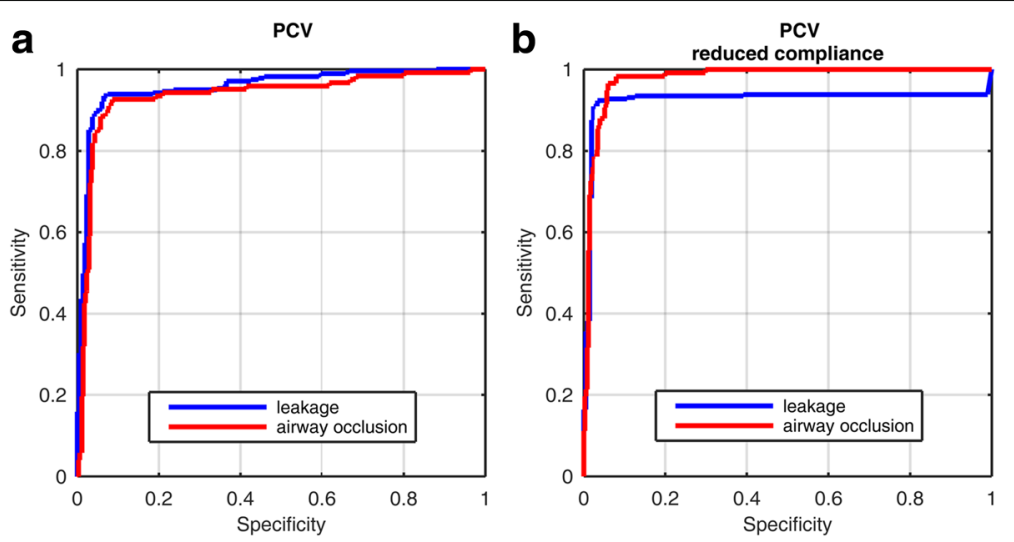

Fig. 5 Receiver operating characteristic (ROC) curves concerning recognition of the two interventions during PCV: controlled leakage (blue line) and simulated airway occlusion (red line) with unrestricted lung compliance (a) and with reduced lung compliance (b) induced by weights (14 kg) placed on the chest

Recently, Nitzschke et al. tested the effect of a CPR assist device with an AED synchronised with a commercially available ventilator on the CPR performance of emergency medical staff [19]. They recommended their study device for ALS providers with limited experience only. Instead of using an expensive ventilator for professional medical staff, our intention was to develop a cost effective innovative functional prototype of a PAV to realize a wide distribution in public places and institutions in order to support laypersons. Moreover, an advantage of the turbine used in our study in contrast to the commercially available ventilators is its independency of any kind of additional gas supply.

To assure patient's safety and to avoid possible harm, e.g. gastric insufflation, the PAV-system has to be able to recognize and properly manage potential complications such as leakage or the status when airways are closed.

As $\mathrm{PetCO}_{2}$ may be low under resuscitation conditions $[20,21]$, in this study we used a mathematical algorithm for the detection of airway occlusion which was based on $\mathrm{P}_{\text {insp }}$ and did not include $\mathrm{PetCO}_{2}$ as a crucial parameter. Compared to medical observation that was based on $\mathrm{PetCO}_{2}, \mathrm{FO}_{2}, \mathrm{P}_{\text {insp }}$ and flow, the sensor system was able to detect leakage and airway occlusion with high sensitivity and specificity over $92 \%$. As a fundamental

Table 3 Results from receiver operating characteristics

\begin{tabular}{lll}
\hline Manoeuvers & $\begin{array}{l}\text { Sensitivity } \\
{[\%]}\end{array}$ & $\begin{array}{l}\text { Specificity } \\
{[\%]}\end{array}$ \\
\hline PCV with controlled leakage & 93.5 & 93.5 \\
PCV with simulated airway occlusion & 91.8 & 91.7 \\
PCV with leakage, reduced lung compliance & 92.4 & 96.6 \\
PCV with airway occlusion, reduced lung & 96.6 & 94.0 \\
compliance & & \\
\hline
\end{tabular}

$\overline{P C V}$ pressure controlled ventilation requirement for support systems used by trained laypersons the sensor prototype could detect whether the airways were closed. This is a necessary prerequisite for immediate interruption of ventilation in those cases. In addition, head position could be monitored by the PAV by means of the 3-axis accelerometer. Thereby, users could be told to perform a head tilt/chin lift manoeuver to open the airways.

In this setting we tried to simulate elevated intrathoracic pressures and reduced lung compliance which may occur when chest compressions are performed during resuscitation [22-24]. As we used pressure controlled ventilation with constant $p_{\text {insp }}$ and constant respiratory rate, significantly reduced lung compliance could have led to decreased tidal volumes and minute ventilation volumes. In contrast to what should have been expected, tidal volumes remained constant when weight was placed onto volunteers' chests and sensitivity and specificity for recognition of airway closure and leakage was even better in this setting. Possible explanations for these unexpected findings could be that primarily thoracic and not lung compliance was reduced when weight was put onto volunteers' chests [25]. In addition, participants may involuntarily have compensated the reduced thoracic compliance through increased (spontaneous) breathing efforts.

Generally, both volume and pressure controlled ventilation can be used in emergency respirators. In case of pressure controlled ventilation a strict monitoring of ventilation pressure is mandatory to avoid gastric air insufflation. As airway pressure $>2.0 \mathrm{kPa}$ causes opening of the oesophageal sphincter, we limited the maximum inspiratory pressure to $1.5 \mathrm{kPa}$ in this study.

Another crucial problem in mask ventilation is leakage occurring when a tight fit of the mask cannot be 
achieved for any reason. As the PAV is to be used by trained laypersons it is highly probable that leakage will occur [4]. Leakage in ventilation is defined as the difference between inspiratory and expiratory tidal volumes. The PAV, therefore, analysed the difference between inspiratory and expiratory flows. This data was then used to determine the percentage of leakage with respect to inspired tidal volume. For non-invasive mask ventilation, commercially available ventilation systems can compensate more than $100 \%$ of leakage. In our study, we used a small, standardized and reproducible leakage of $25-35 \%$ regarding inspired tidal volume. Although this leakage could easily be compensated through the turbine and it did not significantly impair ventilation, the pre-stage PAV reliably recognized the expiratory decrease of tidal volume. For use in emergency settings, leakage recognition could be used together with 3-axis accelerometer output to guide users to readjust mask fitting and head position.

An important limitation of this study was the fact that the pre-stage PAV could not be tested in a real resuscitation situation. The study setup in volunteers did not allow a combination of ventilation and chest compressions. Furthermore, healthy volunteers cannot be regarded as patients with respect to unimpaired spontaneous breathing, protective functions as well as lung compliance. Even a pig model, which is often used as nearly equivalent to human organism had to be excluded due to the different anatomy concerning the epiglottis [26].

Prospectively, sensor data (flow, pressure, $\mathrm{FO}_{2}, \mathrm{FCO}_{2}$, 3-axis accelerometer) could be used for an adapted feedback algorithm [27] integrated in a PAV to guide laypersons in an audio-visually controlled way comparable to well-established automatic external defibrillators (AED) with voice instructions [3, 12].

A potential area for future investigation could be optimization and miniaturization of the sensor system and adaption of the algorithm. In the next step, cardiopulmonary resuscitation studies in life support training manikins supported by optimized PAV are necessary, followed by field studies in patients. In this context, an appropriate teaching and training of BLS providers has to be established sustainably [28].

\section{Conclusion}

In conclusion, the pre-stage PAV provides a respectable basis for the development of an automatic emergency respirator. Evaluation of ventilation parameters such as tidal volume, breathing frequency and inspiratory pressure indicated effective ventilation by the examined device. Safety aspects like leakage and airway occlusion were detected effectively.

\section{Additional files}

\begin{abstract}
Additional file 1: Figure S1. Sensor outputs during PCV manoeuvre under reduced lung compliance. Typical curves for flow, pressure, $\mathrm{FO}_{2}$ and $\mathrm{FCO}_{2}$ are presented over one minute from one healthy volunteer (blue line $=$ flow $[\mathrm{l} / \mathrm{min}]$; red line $=$ pressure $[\mathrm{kPa}]$; green line $=\mathrm{FO}_{2}[\%]$; magenta line $\left.=\mathrm{FCO}_{2}[\%]\right)$. (PDF $1133 \mathrm{~kb}$ )
\end{abstract}

Additional file 2: Figure S2. Sensor outputs during PCV under reduced lung compliance and controlled leakage. According to the study protocol curves for flow, pressure, $\mathrm{FO}_{2}$ and $\mathrm{FCO}_{2}$ were recorded over one minute from one healthy volunteer (black dashed lines = start and stop of controlled leakage; blue line = flow $[\mathrm{l} / \mathrm{min}]$; red line = pressure $[\mathrm{kPa}]$; green line $=\mathrm{FO}_{2}[\%]$; magenta line $=\mathrm{FCO}_{2}[\%]$ ). (PDF $\left.1127 \mathrm{~kb}\right)$

Additional file 3: Figure S3. Sensor outputs during PCV under reduced lung compliance and simulated airway occlusion. Sensor curves for flow, pressure, $\mathrm{FO}_{2}$ and $\mathrm{FCO}_{2}$ are presented over one minute from one healthy volunteer (black dashed lines = start and stop of simulated airway occlusion; blue line $=$ flow $[\mathrm{l} / \mathrm{min}]$; red line $=$ pressure $[\mathrm{kPa}]$; green line $=\mathrm{FO}_{2}[\%]$; magenta line $=\mathrm{FCO}_{2}[\%]$ ). (PDF $1082 \mathrm{~kb}$ )

\section{Abbreviations \\ AED: Automated external defibrillator; BLS: Basic life support; BMI: Body mass index; CPR: Cardio-pulmonary resuscitation; ERC: European Resuscitation Council; $\mathrm{ETCO}_{2}$ : End tidal fraction of carbon dioxide; $\mathrm{FCO}_{2}$ : Carbon dioxide fraction; $\mathrm{F}_{\mathrm{i}} \mathrm{O}_{2}$ : Inspiratory oxygen fraction; Flow ${ }_{\text {insp: Inspiratory flow; }}$ $\mathrm{FO}_{2}$ : Oxygen fraction; l:E: Ratio of inspiration to expiration; MEMS: Micro electro mechanical system; NDIR: Non-dispersive infrared absorption; PAV: Public access ventilator; PCV: Pressure controlled ventilation; PEEP: Positive end expiratory pressure; Peff: Effective pressure; Pinsp: Inspiratory pressure; ROC: Receiver operating characteristics; RR: Respiratory rate; SD: Standard deviation; $V_{\text {exp }}$ : Expiratory volume; VF: Ventricular fibrillation; $V_{\text {insp }}$ : Inspiratory volume}

\section{Acknowledgements}

The authors would like to thank the volunteers for their participation. The study was supported by a joint research project grant from the Ministry of Education Science and Culture Mecklenburg/Norpommern, Germany (V-630-S-111-2011/054 and V-630-S-111-2011/055). The funding sources played no direct role in the planning, conduct, or analysis of the study.

\section{Funding}

This work was supported by a joint research project grant from the Ministry of Education Science and Culture Mecklenburg/Vorpommern, Germany (V-630-S-111-2011/054 and V-630-S-111-2011/055). The funding organization had no involvement in the study design, in the collection, analysis and interpretation of data, in the writing of the manuscript or in the decision to submit the manuscript for publication.

\section{Availability of data and materials}

The complete dataset supporting the findings of this article is available from the corresponding author on request.

\section{Authors' contributions}

PF, JKS, HE, and JS conceived the study and designed the experiments; PF, $\mathrm{HN}$, and $\mathrm{JO}$ undertook recruitment of volunteers and SK as a physician observed the conduct of experiments; $\mathrm{HN}, \mathrm{HJ}, \mathrm{MB}, \mathrm{PF}$, and $\mathrm{JO}$ collected and managed the data, including quality control; MD, PF, JO, and SK analysed the data, conducted the statistical analyses and interpreted the data; PF, JO, SK, and JKS drafted the manuscript, and all authors contributed substantially to its revision and PF takes responsibility for the paper as a whole. All authors read and approved the final manuscript.

Ethics approval and consent to participate

Ethical approval was obtained by the ethics committee from Rostock University Medical Centre (approval No: A 2014-0015). All 52 participants were informed about the purpose of the study and gave written informed consent. 


\section{Consent for publication}

Written informed consent for the publication from the participants enrolled in this study may be provided on request. Patient consented for Fig. 1 to be published in the manuscript.

\section{Competing interests}

Sensatronic GmbH (Mr. Jens Schwarz) is manufacturer and distributor for several sensor parts and the device described in the manuscript. For all other authors conflicts of interest do not exist.

\section{Publisher's Note}

Springer Nature remains neutral with regard to jurisdictional claims in published maps and institutional affiliations.

\section{Author details \\ ${ }^{1}$ Department of Anaesthesiology and Intensive Care Medicine, Rostock University Medical Centre, Schillingallee 35, 18057 Rostock, Germany. ${ }^{2}$ Institute for General Electrical Engineering, University of Rostock, 18059 Rostock, Germany. ${ }^{3}$ Sensatronic GmbH Wismar, 23970 Wismar, Germany.}

\section{Received: 14 July 2017 Accepted: 23 November 2017}

Published online: 04 December 2017

\section{References}

1. Gräsner J-T, Bossaert L. Epidemiology and management of cardiac arrest: what registries are revealing. Best Pract Res Clin Anaesthesiol. 2013;27:293-306.

2. Nolan JP, Perkins GD, Soar J. Improving survival after out-of-hospital cardiac arrest. BMJ. 2015:h4989.

3. Hanefeld CA. First city-wide early defibrillation project in a German city: 5year results of the Bochum against sudden cardiac arrest study. Scand Trauma Resusc Emerg Med. 2010;18:31.

4. Perkins GD, Handley AJ, Koster RW, Castrén M, Smyth MA, Olasveengen T, et al. European resuscitation council guidelines for resuscitation 2015: section 2. Adult basic life support and automated external defibrillation. Resuscitation. 2015;95:81-99.

5. Souchtchenko SS, Benner JP, Allen JL, Brady WJA. Review of chest compression interruptions during out-of-hospital cardiac arrest and strategies for the future. J Emerg Med. 2013;45:458-66.

6. Cha K-C, Kim YW, Kim TH, Jung WJ, Yook H, Choi E, et al. Comparison between 30:1 and 30:2 compression-to-ventilation ratios for cardiopulmonary resuscitation: are two ventilations necessary? Acad Emerg Med. 2015;22:1261-6.

7. Nichol G, Leroux B, Wang H, Callaway CW, Sopko G, Weisfeldt M, et al. Trial of continuous or interrupted chest compressions during CPR. N Engl J Med. 2015:373:2203-14.

8. Sasson C, Rogers MAM, Dahl J, Kellermann AL. Predictors of survival from out-of-hospital cardiac arrest: a systematic review and meta-analysis. Circ Cardiovasc Qual Outcomes. 2010;3:63-81.

9. Locke CJ. Bystander cardiopulmonary resuscitation: concerns about mouthto-mouth contact. Arch Intern Med. 1995;155:938.

10. Bobrow BJ, Spaite DW, Berg RA, Stolz U, Sanders AB, Kern KB, et al. Chest compression-only CPR by lay rescuers and survival from out-of-hospital cardiac arrest. JAMA. 2010;304:1447.

11. Hüpfl M, Selig HF, Nagele P. Chest-compression-only versus standard cardiopulmonary resuscitation: a meta-analysis. Lancet. 2010;376:1552-7.

12. Nielsen AM, Folke F, Lippert FK, Rasmussen LS. Use and benefits of public access defibrillation in a nation-wide network. Resuscitation. 2013;84:430-4

13. Waalewijn RA, Tijssen JG, Koster RW. Bystander initiated actions in out-ofhospital cardiopulmonary resuscitation: results from the Amsterdam resuscitation study (ARRESUST). Resuscitation. 2001;50:273-9.

14. Perkins GD, Jacobs IG, Nadkarni VM, Berg RA, Bhanji F, Biarent D, et al. Cardiac arrest and cardiopulmonary resuscitation outcome reports: update of the Utstein resuscitation registry templates for out-of-hospital cardiac arrest. Resuscitation. 2015;96:328-40.

15. Wenzel V, Lehmkuhl P, Kubilis PS, Idris AH, Pichlmayr I. Poor correlation of mouth-to-mouth ventilation skills after basic life support training and 6 months later. Resuscitation. 1997:35:129-34.

16. Spooner BB, Fallaha JF, Kocierz L, Smith CM, Smith SCL, Perkins GD. An evaluation of objective feedback in basic life support (BLS) training. Resuscitation. 2007;73:417-24
17. Woollard M, Smith A, Whitfield R, Chamberlain D, West R, Newcombe R, et al. To blow or not to blow: a randomised controlled trial of compressiononly and standard telephone CPR instructions in simulated cardiac arrest. Resuscitation. 2003:59:123-31.

18. Kellermann AL, Hackman BB, Somes G. Dispatcher-assisted cardiopulmonary resuscitation. Validation of efficacy. Circulation. 1989:80:1231-9.

19. Nitzschke R, Doehn C, Kersten JF, Blanz J, Kalwa TJ, Scotti NA, et al. Effect of an interactive cardiopulmonary resuscitation assist device with an automated external defibrillator synchronised with a ventilator on the CPR performance of emergency medical service staff: a randomised simulation study. Scand J Trauma Resusc Emerg Med. 2017:25.

20. Hartmann SM, Farris RWD, Di Gennaro JL, Roberts JS. Systematic review and meta-analysis of end-tidal carbon dioxide values associated with return of spontaneous circulation during cardiopulmonary resuscitation. J Intensive Care Med. 2015:30:426-35.

21. Grmec S, Klemen P. Does the end-tidal carbon dioxide (EtCO2) concentration have prognostic value during out-of-hospital cardiac arrest? Eur J Emerg med off J Eur Soc. Emerg Med. 2001;8:263-9.

22. Ornato JP, Bryson BL, Donovan PJ, Farquharson RR, Jaeger C. Measurement of ventilation during cardiopulmonary resuscitation. Crit Care Med. 1983;11:79-82.

23. Davis K, Johannigman JA, Johnson RC, Branson RD. Lung compliance following cardiac arrest. Acad Emerg med off J Soc. Acad Emerg Med. 1995;2:874-8

24. Johannigman JA, Branson RD, Davis K, Hurst JM. Techniques of emergency ventilation: a model to evaluate tidal volume, airway pressure, and gastric insufflation. J Trauma. 1991;31:93-8.

25. Gabrielli A, Layon AJ, Idris AH. Physiology of ventilation during cardiac arrest. In: Ornato JP, Peberdy MA, editors. Cardiopulmonary Resuscitation. Totowa: Humana Press; 2005. p. 39-94.

26. Wolcke B. Laienreanimation - Kontroversen in der Notfallmedizin: mit oder ohne Beatmung? AINS - Anästhesiol · Intensivmed · Notfallmedizin Schmerzther. 2013:48:558-561.

27. Fuchs $P$, Obermeier J, Kamysek S, Degner M, Nierath $H$, Jürß H, et al. Public access ventilator - a functional prototype for emergency ventilation by trained layperson. Resuscitation. 2015;96:50.

28. Jorge-Soto C, Abelairas-Gómez C, Barcala-Furelos R, Garrido-Viñas A Navarro-Patón R, Muiño-Piñeiro M, et al. Automated external defibrillation skills by naive schoolchildren. Resuscitation. 2016;106:37-41.

\section{Submit your next manuscript to BioMed Central and we will help you at every step:}

- We accept pre-submission inquiries

- Our selector tool helps you to find the most relevant journal

- We provide round the clock customer support

- Convenient online submission

- Thorough peer review

- Inclusion in PubMed and all major indexing services

- Maximum visibility for your research

Submit your manuscript at www.biomedcentral.com/submit
Biomed Central 